\title{
Experimental verification of an Oseen flow slender body theory
}

\author{
E. CHADW ICK ${ }^{1} \dagger$, H. M. KHAN ${ }^{1} \ddagger$, \\ M. MOATAMEDI ${ }^{2}$, M. MAPPIN ${ }^{1}$ AND M. PENNEY ${ }^{1}$ \\ ${ }^{1}$ School of Computing, Science and Engineering, University of Salford, Salford M5 4WT, UK \\ ${ }^{2}$ Narvik University College, Lodve Langes Gate 2, Narvik, N-8505 Norway
}

(Received 8 September 2009; revised 10 March 2010; accepted 11 March 2010; first published online 25 May 2010)

Consider uniform flow past four slender bodies with elliptical cross-section of constant ellipticity along the length of $0,0.125,0.25$ and 0.375 , respectively, for each body. Here, ellipticity is defined as the ratio of the semiminor axis of the ellipse to the semimajor axis. The bodies have a pointed nose which gradually increases in cross-section with a radius of curvature $419 \mathrm{~mm}$ to a mid-section which then remains constant up to a blunt end section with semimajor axis diameter $160 \mathrm{~mm}$, the total length of all bodies being $800 \mathrm{~mm}$. The bodies are side-mounted within a low-speed wind tunnel with an operational wind speed of the order $30 \mathrm{~m} \mathrm{~s}^{-1}$. The side force (or lift) is measured within an angle of attack range of $-3^{\circ}$ to $3^{\circ}$ such that the body is rotated about the major axis of the ellipse cross-section. The lift slope is determined for each body, and how it varies with ellipticity. It is found that this variance follows a straight line which steadily increases with increasing ellipticity. It is shown that this result is predicted by a recently developed Oseen flow slender body theory, and cannot be predicted by either inviscid flow slender body theory or viscous crossflow theories based upon the Allen and Perkins method.

\section{Introduction}

Slender body theories in fluid flow have been developed over the last 80 years, starting with Munk (1924) who used the inviscid flow approximation to model airship manoeuvring. Jones (1945) also used a slenderness assumption to accurately model the lift on a dart delta wing using inviscid flow theory. However, for bodies with fat sections, such as those for a missile or a ship, discrepancies occur for the lift force which are noted by Allen and Perkins for missiles (Allen \& Perkins 1951) and Newman for ships (Newman 1977). Both give the experimental lift force to be much greater than that given by inviscid flow theory, and Allen and Perkins suggest an additional viscous crossflow force to be added onto the force predicted by inviscid flow theory, and which is calculated from experimental crossflow data producing a semiempirical method. This approach is the basis of the currently and widely used United States Air Force code Missile Datcom (Simon \& Blake 1999). Allen and Perkins

$\ddagger$ H. M. Khan is also a permanent faculty member at College of Aeronautical Engineering, National University of Sciences and Technology (NUST), Pakistan

$\dagger$ Email address for correspondence: e.a.chadwick@salford.ac.uk 
only considered bodies with circular cross-section, but Jorgensen (1957) suggested an extension for bodies with elliptical cross-section by using Newtonian impact theory.

Recently, Chadwick suggested evaluating the additional viscous lift force by using Oseen flow theory (Chadwick 2009) (based on the Oseen velocity expansion in terms of Oseenlets, Chadwick 1998; and the slender body theory in Oseen flow, Chadwick 2002), thereby eliminating the requirement to evaluate it empirically. This theory still assumes that along the body length the flow is approximately inviscid and so the slip boundary condition can be applied, but it additionally requires that a far-field Oseen flow wake develops downstream. In the following paragraph, a physical explanation of the theory is given, including a discussion on the particular choice of boundary conditions and governing equations. This details the significant points given in greater depth in the papers Chadwick (2002), Chadwick \& Fishwick (2007) and Chadwick (2009).

The overall aim is to model the underlying incompressible Navier-Stokes flow sufficiently accurately that the resulting lift equation has close agreement with experiment. There are certain regions of fluid where we expect the Navier-Stokes flow to be approximated closely by simpler flow descriptions, namely in the far-field region, and also in the fluid region over the length of the body. In the far-field, we expect Oseen flow to provide a good approximation and we know that the Oseen linearization is sufficient to evaluate the forces. The properties of this flow are the development of a wake region of vorticity and viscosity (or retarded momentum) and outside of this wake an approximately inviscid flow. Similarly, over the length of a streamlined slender body for which the boundary layer remains attached, the flow is expected to be approximately inviscid up to the boundary layer. However, there are regions of the fluid which are not modelled by the slender body theory tested in this paper, namely the boundary layer and the near wake. A complete description would include these. In the near-wake region, relatively still water resides near the base at the rear of the body exerting a longitudinal pressure force along the length of the body. Therefore, the component in the direction normal to the body axis (the normal force or side force) is negligible. Consequently, for small angles of attack the contribution to the lift force (which is measured normal to the free stream velocity) from the near wake will also be negligible; in the present paper we consider the angle of attack up to $3^{\circ}$ only. Including the boundary layer would mean applying the no-slip boundary condition. However, the boundary layer thickness is negligible and so its effect is not expected to substantially change the inviscid flow description along the body length. The solution of the inviscid flow over the length of the body can be obtained within a slender body framework by applying the slip boundary condition together with the Kutta condition assumption that flow leaves smoothly at the end section. Furthermore, we expect from the above arguments that the far-field inviscid flow term which produces lift is obtained from this solution and can be matched to the inviscid potential within the far-field Oseen flow term which produces lift. The Oseen flow term producing lift is called the lift Oseenlet and produces unit lift such that exactly half of the lift originates from the inviscid part and exactly half from the viscous wake part. This ratio holds irrespective of the Reynolds number, and so holds in the high Reynolds number limit or equivalently as the viscosity tends to zero. For this reason, the lift contribution from the term in the Oseen flow representation of viscous origin is not Reynolds number dependent, and so the lift formulation does not contain the viscous coefficient and is not merely a geometry effect within a framework of a purely inviscid flow theory. We note that the vortex wake induces pressure changes 
along the body surface, and these are captured through the matching: the vortex wake is modelled by an integral distribution of lift Oseenlets which are equivalent to infinitesimal horseshoe vortices (Chadwick 2005); slender body theory is then applied from this model to determine the pressure changes at a cross-section along the body length (Chadwick 2002).

This theory enables the additional viscous lift force to be evaluated implicitly within the theory, unlike the Allen and Perkins method (Allen \& Perkins 1951) which evaluates it explicitly by a semi-empirical method. Fishwick (2005) compared a wide range of NACA missile experiments for lift force against both the Allen and Perkins method and also the Oseen flow theory of Chadwick, and found good agreement between them all.

However, for bodies with thin rather than circular cross-section, there are significant discrepancies between viscous crossflow theories and the new Oseen flow theory of Chadwick. Such bodies are also of practical importance in both missile and ship design, as thinner cross-sections can give improved manoeuvring performance. Unfortunately, because convention is for data to be presented for the lift coefficient involving a division by base area, for bodies of cross-sectional area close to zero it is difficult to draw conclusions about the Oseen theory from current experimental studies on slender bodies (Sigal 1991).

In the present paper, we devise an experimental test for the new theory to predict the lift force for bodies of thin cross-section. Four bodies are built, with ellipticity 0 , $0.125,0.25$ and 0.375 , respectively. The semimajor axis length (and not cross-sectional area) is kept constant across the models, which then enables the new theory to be tested. Theory predicts a straight line increase with increasing ellipticity for the lift slope (which is the gradient of the lift line plotted against angle of attack). The experiments are conducted in a low-speed wind tunnel, and are shown to follow this straight line variation. Neither inviscid flow theory nor the viscous crossflow methods are able to predict this.

\section{Models}

All models have a total length of $800 \mathrm{~mm}$ and major axis width $160 \mathrm{~mm}$. Each model has a front nose section in the first $250 \mathrm{~mm}$ with a radius of curvature of $419 \mathrm{~mm}$. From the middle to the end section the models have a constant elliptical cross-section (see figure 1). Hence, the slenderness ratio is similar to that used by Jones (1945) and so for bodies of thin cross-section the slenderness assumption is expected to hold. The first model with ellipticity zero (flat plate) is made out of sheet metal $3 \mathrm{~mm}$ thick, with the appropriate profile drawn and cut out. The other three models with ellipticity $e$ of $0.125,0.25$ and 0.375 respectively are first created as computer-aided design (CAD) drawings and then sent to a three-dimensional printer which makes the models out of acrylonitrile butadiene styrene (ABS) thermoplastic. To provide additional structural strength, a slit is made in the models within which is inserted an aluminium plate $3 \mathrm{~mm}$ thick. The two side supports positioned at $260 \mathrm{~mm}$ and $340 \mathrm{~mm}$ from the nose respectively also screw into this plate. Once the models have been made, the front tips of all are rounded by sanding down to reduce vortex shedding from the nose as far as possible. Rear (sting) mounting was also tried but caused vibration of the model and so this approach was abandoned, although the results for lift (or side) force were not noticeably different from the equivalent results using side mounting. 


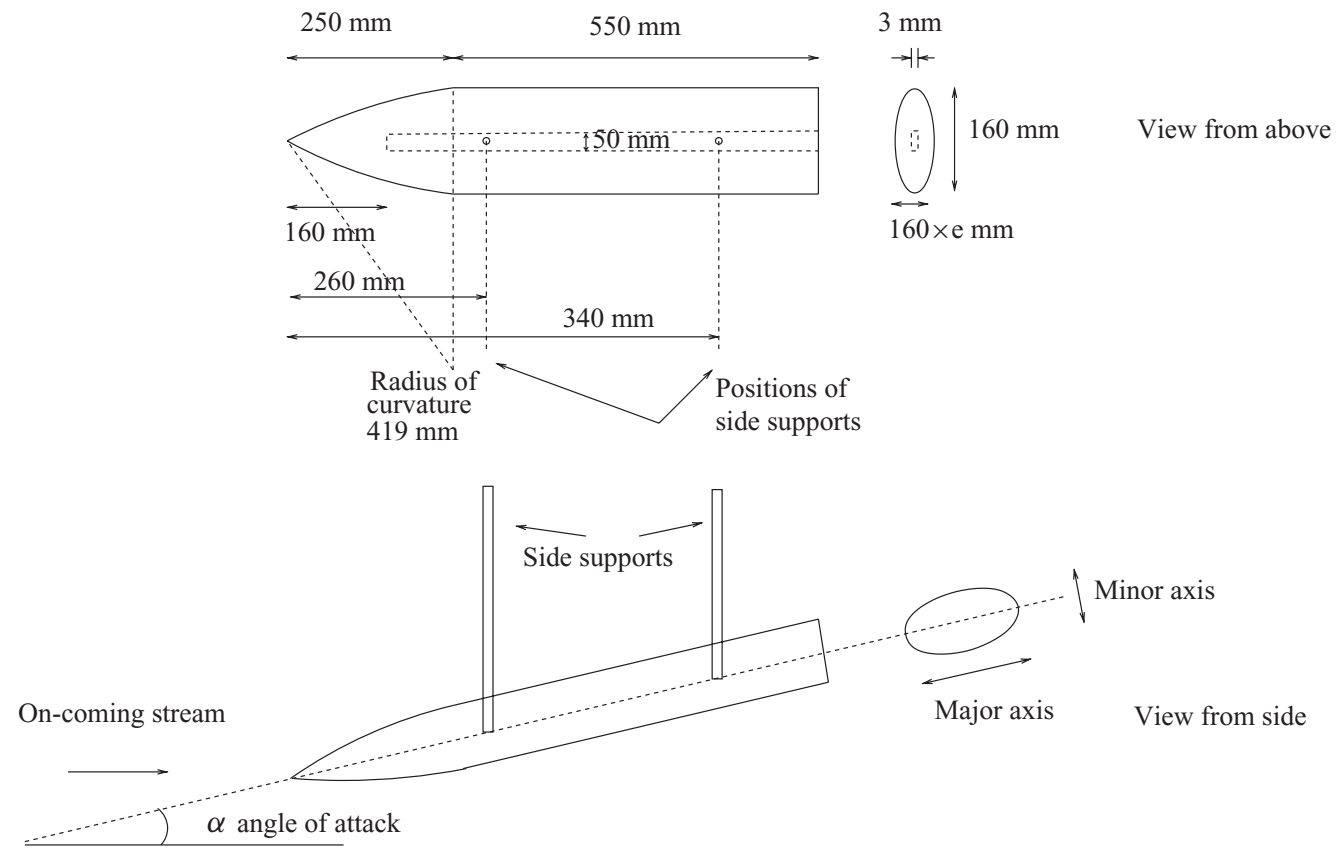

FIGURE 1. The model dimensions.

\section{Wind tunnel}

A closed-circuit wind tunnel is used, with a cuboid working section of dimensions $1800 \mathrm{~mm} \times 1150 \mathrm{~mm} \times 850 \mathrm{~mm}$ width. The wind tunnel has a working wind speed of the order $30 \mathrm{~m} \mathrm{~s}^{-1}$. The correction due to downwash effects is negligible because of the small slender body width and small angle of attack range. Also, the relative symmetry in the lift results suggests that the flow disturbance due to the mounts is negligible. The model dimensions are chosen to maximize the allowable length within the working section and allowable width for which slender body theory is applicable, in order to get a sufficiently strong load response recorded by the load balance in the angle of attack range. The angle of attack range was chosen sufficiently small so as to minimize any uplift due to vortex shedding along the length.

\section{Results}

The standard presentation of results for slender bodies is by normalizing the lift to obtain a lift coefficient, which is the lift divided by the multiple of the base area with the free-stream dynamic pressure. However, because the base area is small for slender bodies with thin section, it is difficult to then interpret the results. Instead, we shall introduce a different lift coefficient which takes its lead from Lighthill's result for lift from inviscid flow theory (Lighthill 1960). Lighthill notes that the inviscid lift remains approximately the same if the circle circumference that exactly encompasses the end section has the same radius regardless of the shape. We suggest that a more meaningful lift coefficient useful for comparisons would therefore use the cross-sectional area of this circle that circumscribes the end section rather than the base area. For our models, this is $\pi s^{2}$ where $s$ is the semimajor axis cross-sectional length. The results presented in figures $2-5$ for lift (or side) force coefficient against 
Ellipticity 0

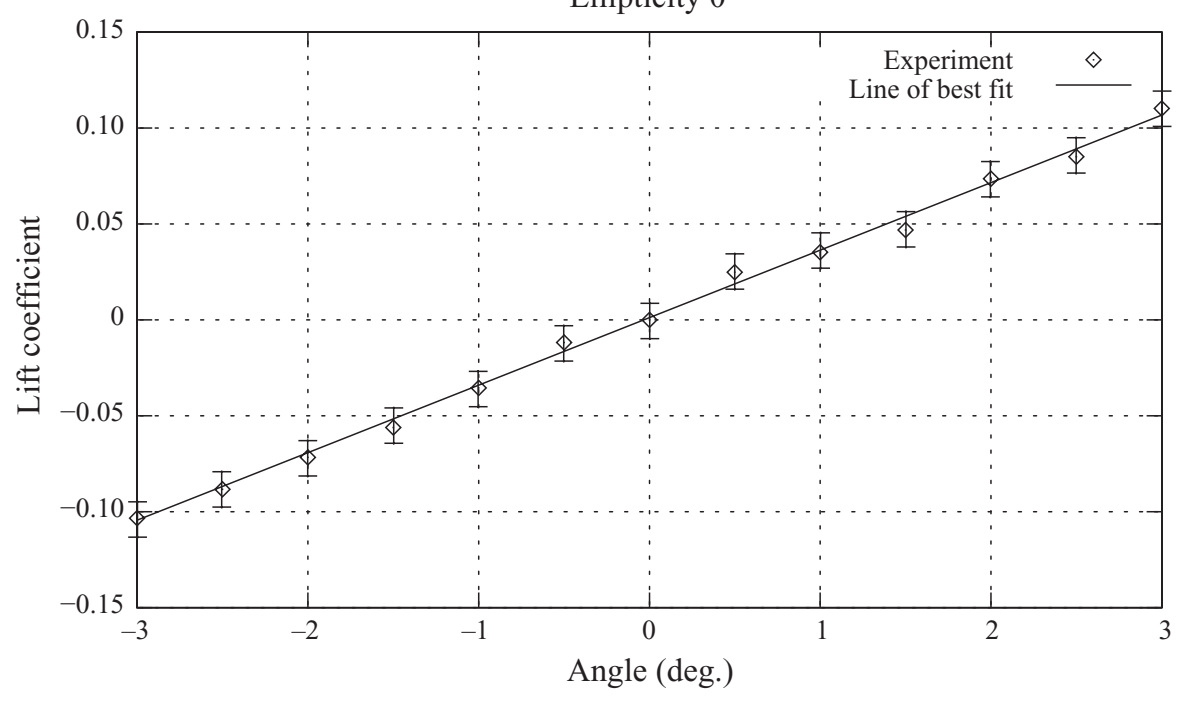

FIGURE 2. Lift force over angle range $-3^{\circ}$ to $3^{\circ}$ for ellipticity 0 .

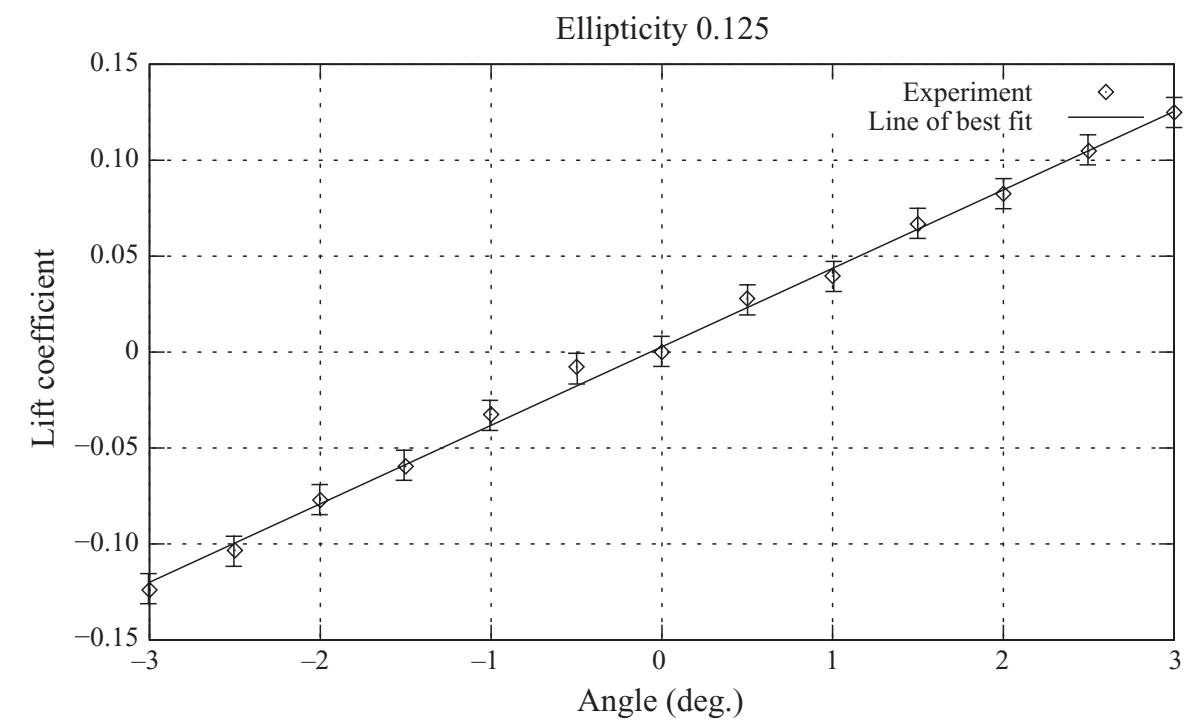

Figure 3. Lift force over angle range $-3^{\circ}$ to $3^{\circ}$ for ellipticity 0.125 .

angle of attack were obtained for the four models in the range of $-3^{\circ}$ to $3^{\circ}$. When repeating the experiments, it was found that the relative error lay within the range \pm 0.01 for the lift coefficient value. The results are plotted for a single run, including error bars relating to this variation. A line of best fit using the standard least squares fit algorithm is also plotted.

\section{Comparison}

Using these experimental results, we can now make a comparison between experiment, slender body inviscid theory, Chadwick's Oseen based flow theory 


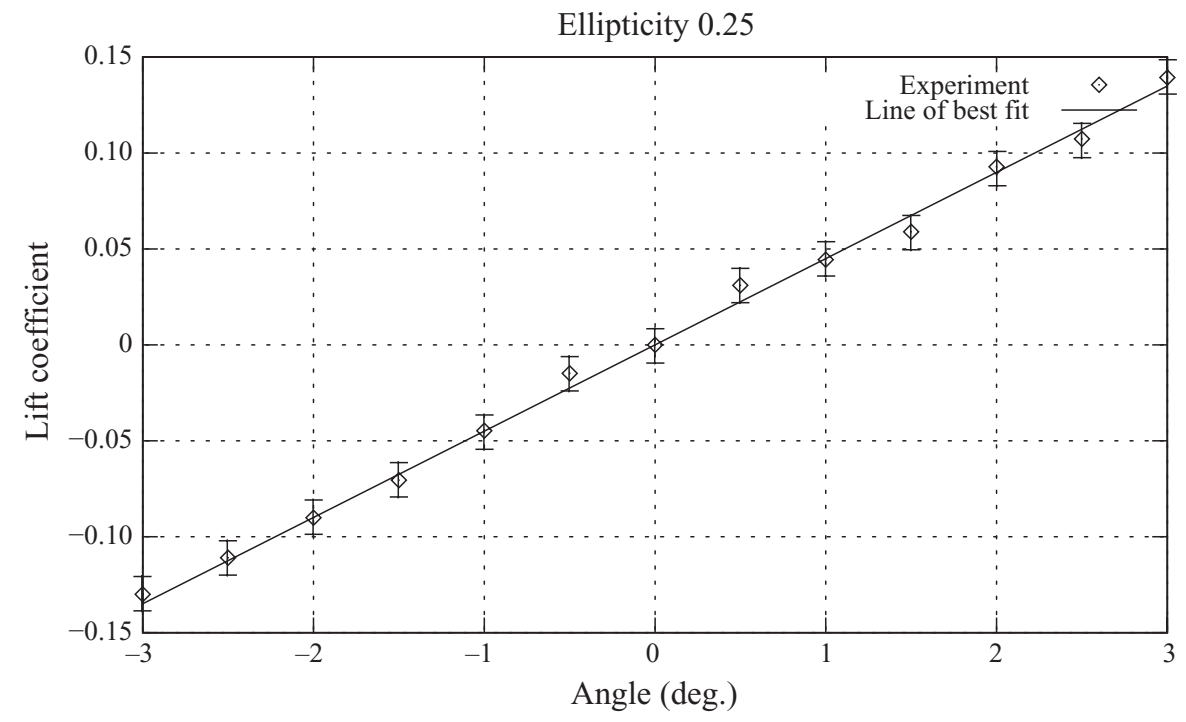

Figure 4. Lift force over angle range $-3^{\circ}$ to $3^{\circ}$ for ellipticity 0.25 .

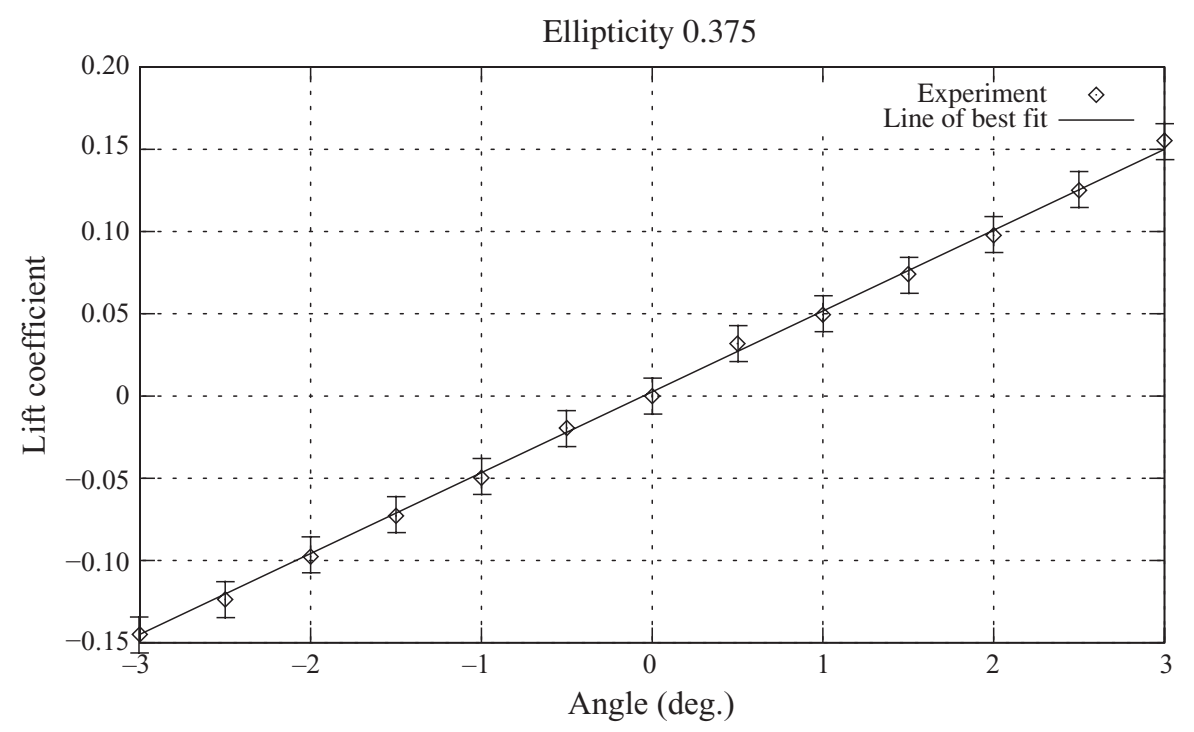

FIGURE 5. Lift force over angle range $-3^{\circ}$ to $3^{\circ}$ for ellipticity 0.375 .

(Chadwick \& Fishwick 2007) and Jorgensen's extension to viscous crossflow theory for slender bodies with elliptical cross-section for which he gives a means of evaluation for different ellipticity in Jorgensen (1973). It is noted that although (5.1)-(5.4) give the lift in terms of parameters at the end section only, this does not imply they are only for bodies of uniform cross-section. It is a result of slender body theory that the lift is given in terms of the end section only as long as the body is sufficiently slender. This means that the slender body profile leading up to the end section does not influence the lift in theory, although the theory works best for streamlined bodies tapering to a point at the nose. 


$\begin{array}{lc}\text { Ellipticity } & \text { Per cent difference } \\ 0.707 & 5 \\ 0.667 & 6 \\ 0.5 & 12 \\ 0.333 & 23 \\ 0.25 & 31 \\ 0.2 & 37 \\ 0.167 & 41\end{array}$

TABLE 1. Jorgensen's prediction (Jorgensen 1973) for the lift (or side) force.

\subsection{Slender body inviscid flow theory}

This result has been given in many places, perhaps the most easy to use form given by Lighthill (1960). This gives the lift (or side force) $L$ for a slender body with elliptical cross-section in inviscid flow as

$$
L=\pi s^{2} \rho U^{2} \alpha
$$

and a lift coefficient of

$$
C_{L}=\frac{\pi s^{2} \rho U^{2} \alpha}{\pi s^{2}(1 / 2) \rho U^{2}}=2 \alpha .
$$

We denote the density by $\rho$, uniform stream velocity $U$ and angle of attack $\alpha$ in radians.

\subsection{Slender body Oseen flow theory}

The Oseen flow theory of Chadwick \& Fishwick (2007) gives the lift for a body with elliptical cross-section inclined at angle of attack by rotating about the major elliptical axis as

$$
L=\pi s^{2} \rho U^{2} \alpha(1+e)
$$

and a lift coefficient $C_{L}$ of

$$
C_{L}=2 \alpha(1+e),
$$

where the ellipticiy is denoted by $e$, and is defined as the ratio of the semimajor axis of the ellipse to the semiminor axis.

\subsection{Jorgensen's viscous crossflow Newtonian impact theory}

Jorgensen (1973) gives the per cent difference between his slender body Newtonian based theory and standard inviscid slender body theory for the ratio between the lift at elliptical cross-section to the lift at circular cross-section. This is given, with the ellipticity, in the form of a table and is reproduced here.

We also note that for ellipticity 0 , the corresponding percentage difference given by Jorgensen is 100 . Let us assume a lift value of close to $L=2 \pi s^{2} \rho U^{2} \alpha$ for a body with circular cross-section radius $s$, which is consistent with results expected from Allen and Perkins theory and also from experiment by Fishwick (2005). Then, using the table 1 we can determine the predicted lift from Jorgensen for bodies with elliptical cross-section and presented in figure 6 by reducing this amount by the corresponding percentage given in the table. On the vertical axis is plotted the lift slope which we give to be $L /\left(\pi s^{2}(1 / 2) \rho U^{2} \alpha\right)$ or $C_{L} / \alpha$, and on the horizontal axis is plotted the ellipticity $e$. The values for the lift coefficient slope are obtained from the gradient of 


$\begin{array}{lcc}\text { Ellipticity } & \text { Experiment } & \text { Theory } \\ 0 & 2.020 & 2 \\ 0.125 & 2.349 & 2.25 \\ 0.25 & 2.550 & 2.5 \\ 0.375 & 2.848 & 2.75\end{array}$

TABLE 2. Lift coefficent slope values for theory and experiment.

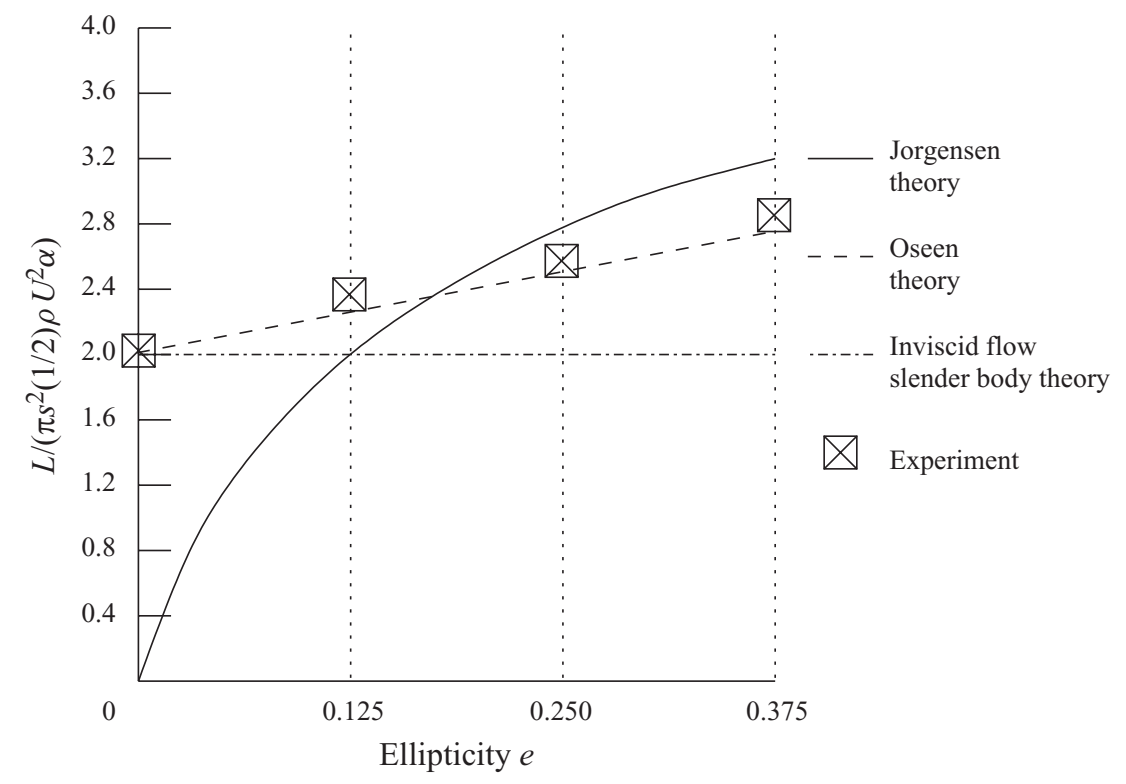

FIGURE 6. Comparison between experiment and theories.

the line of best fit using the standard least squares algorithm. These give the following values in table 2 where the angle measure is given in radians, and then plotted on the graph in figure 6.

We see that the experiments follow a gradually increasing straight line variation which closely follows and is just above the Oseen theory. A possible explanation why the results are slightly above the Oseen theory is due to additional uplift due to vortex shedding along the length. It is also noted that for ellipticity $e=0$, the model was made from a flat metal plate and there is only a one percent difference between theory and experiment. The other models with ellipticity $e \neq 0$ were made from the ABS plastic had a rougher and more ribbed surface finish. This may contribute towards greater vortex shedding along the length and so a more pronounced difference between theory and experiment.

From figure 6, it is apparent that both inviscid flow slender body theory and Jorgensen's extension to viscous crossflow theory do not follow experiment, unlike the Oseen flow slender body theory. The set of experiments follow closely the expected prediction and we conclude that they verify the Oseen flow slender body theory.

We would like to acknowledge EPSRC for providing funding for the above experiments through EPSRC grant number EP/G009309/1 entitled: experimental tests to validate a new slender body theory in steady, incompressible Oseen flow. We 
would like to acknowledge support from the College of Aeronautical Engineering, National University of Sciences and Technology (NUST), Pakistan. On a personal note, we would also like to thank a group of attendees who met at the International Conference on Mathematical Fluid Dynamics at the University of Hyderabad, India in 2004 for their continued support and guidance, in particular, Professor P. N. Shankar, Dr B. S. Padmavati, Professor H. K. Moffat FRS and Professor Y. Fukumoto.

\section{REFERENCES}

Allen, H. J. \& Perkins, E. W. 1951 A study of effects of viscosity on flow over slender inclined bodies of revolution. Tech. Rep. Report 1048. NACA.

Chadwick, E. 1998 The far field Oseen velocity expansion. Proc. R. Soc. A 454, 2059-2082.

Chadwick, E. 2002 A slender-body theory in Oseen flow. Proc. R. Soc. A 458, 2007-2016.

Chadwick, E. 2005 A slender wing theory in potential flow. Proc. R. Soc. A 461, 415-432.

Chadwick, E. 2009 A slender body theory in Oseen flow obtained by expanding the Oseenlets in the Green's integral representation. Fluid Dyn. Res. 41, 045508.

Chadwick, E. \& Fishwick, N. 2007 Lift on slender bodies with elliptical cross-section evaluated by using an Oseen flow model. SIAM. J. Appl. Math 67 (5), 1465-1478.

Fishwick, N. J. 2005 Manoeuvring characteristics of slender bodies through fluid. PhD thesis, University of Salford.

JONES, R. T. 1945 Properties of low-aspect-ratio pointed wings at speeds below and above the speed of sound. Tech. Rep. 835. NACA.

JoRgENSEN, L. H. 1957 Elliptic cones alone and with wings at supersonic speeds. Tech. Rep. 1376. NACA.

Jorgensen, L. H. 1973 Prediction of static aerodynamic characteristics for space-shuttle-like and other bodies at angles of attack from 0 to 180. Tech. Rep. TN D-6996. NASA.

Lighthill, M. J. 1960 Note on the swimming of slender fish. J. Fluid Mech. 9, 305-317.

MunK, M. M. 1924 The aerodynamic forces on an airship hull. Tech. Rep. 184. NACA.

Newman, J. N. 1977 Marine Hydrodynamics. MIT Press.

Sigal, A. 1991 Methods of analysis and experiments for missiles with noncircular fuselages. In Tactical Missile Aerodynamics (ed. M. R. Mendenhall), Ch. 5, pp. 171-223. AIAA.

Simon, J. M. \& Blake, W. B. 1999 Missile Datcom: high angle of attack capabilities. AIAA AIAA-99-4258, 1-11. 